\title{
LA BASURA ELECTRÓNICA Y LA CONTAMINACIÓN AMBIENTAL
}

\author{
Luis Hidalgo Aguilera ${ }^{1}$
}

\begin{abstract}
RESUMEN
En las últimas décadas y principalmente al inicio del nuevo siglo, se ha incrementado en grandes proporciones la fabricación, el consumo y el desecho de aparatos eléctricos y electrónicos, por la gran cantidad de beneficios y facilidades que ha dado al desarrollo de la humanidad.

El presente trabajo tiene por objeto identificar a los principales aparatos eléctricos y electrónicos, sus componentes peligrosos para el medio ambiente y para el ser humano, las razones por las cuales los vuelven peligrosos, las formas de deshacerse una vez que terminan su vida útil y las medidas que se han tomado y que se piensa tomar con este tipo de basura que es ya una seria preocupación a nivel mundial.
\end{abstract}

\section{INTRODUCCIÓN}

La producción y la utilización de aparatos y equipos electrónicos aumenta de manera acelerada a nivel mundial en todos los ámbitos en los que se desenvuelve el ser humano, de ahí que la industria electrónica y principalmente la de producción de aparatos constituye actualmente el sector de mayor crecimiento en los países desarrollados.

Para nadie es desconocido los grandes beneficios que se obtienen de la utilización de estos aparatos electrónicos, pues contribuyen no sólo al desarrollo científico, tecnológico e industrial de una sociedad sino que además le brindan comodidad y seguridad.

Pero así como el aumento del consumo de los aparatos electrónicos ha sido vertiginoso a nivel mundial, así también ha ido aumentando la producción de deshechos de este tipo de productos ocasionado por la sustitución, renovación o eliminación paradójicamente por el desarrollo de los mismos.

\footnotetext{
${ }^{1}$ Facultad de Ciencias de la Ingeniería, Universidad Tecnológica Equinoccial, Av. Occidental y Mariana de Jesús. Quito, Ecuador.lhidalgo@hotmail.com
} 
“Anualmente se generan entre 20 y 50 millones de toneladas de basura electrónica al año" (Martínez, 2008), "se calcula que el volumen de la chatarra electrónica está creciendo entre un $16 \%$ y un $28 \%$ cada cinco años" (Duery, 2007), lo que le convierte a este desperdicio en el de mayor crecimiento en los últimos años.

Ya sean considerados como desecho, como basura, como chatarra o como desperdicio, ya es motivo de preocupación su manejo adecuado de "manera amigable" con el ambiente y segura para los manipuladores al deshacerse de los mismos.

Actualmente uno de los principales y graves problemas es el desechar este tipo de basura, porque la mayoría de aparatos electrónicos contiene elementos tóxicos que al no ser adecuadamente gestionados o tratados en su eliminación producen grandes daños al medio ambiente afectando por lo tanto a todos su componentes y particularmente al ser humano.

\section{METODOLOGÍA}

A través de una recopilación bibliográfica, se busca conocer la situación actual de: los principales aparatos electrónicos que mayormente están siendo utilizados por la humanidad; los tiempos de vida útil o de renovación; su composición e identificación de los elementos peligrosos para el medio ambiente; las formas de deshacerse y las propuestas de parte de Organismos especializados.

\section{APARATOS ELÉCTRICOS Y ELECTRÓNICOS CONVERTIDOS EN DESECHOS}

En la actualidad la línea de separación entre la electrónica y la electricidad es ligera, demasiado pequeña, por no decir nula, por lo tanto de manera general se define como aparato o equipo eléctrico y electrónico a todo aquel que funcione adecuadamente a base de electricidad como fuente de energía. Estos aparatos cuando dejan de ser utilizados porque han cumplido con su ciclo de vida útil para una necesidad determinada, pasan a constituirse como todo en la vida en elementos llamados residuos o desechos cuya nomenclatura en español es RAEE (Residuos de Aparatos Eléctricos y Electrónicos) o en Inglés WEEE (Waste Electrical and Electronical Equippament).

Por todos es conocido que estos aparatos son utilizados tanto a nivel industrial, comercial, educativo, doméstico y personal, pues representan ventajas competitivas y marcan el grado de paridad en su 
avance con el desarrollo tecnológico. Por tanto es necesario identificar a los principales aparatos que con el tiempo se convierten en residuo, pudiéndose mencionar a los siguientes:

1. Tarjetas electrónicas utilizadas en el control industrial.

2. Herramientas eléctricas.

3. Lámparas fluorescentes.

4. Computadores de escritorio.

5. Computadores portátiles.

6. Monitores.

7. Impresoras.

8. Escáneres.

9. Video Cámaras.

10. Equipos de Audio.

11. Televisores.

12. DVD.

13. Juguetes electrónicos.

14. Teléfonos Fijos.

15. Teléfonos Móviles.

16. Electrodomésticos en general.

Basta una simple inspección no tan rigurosa, para darnos cuenta que la gran mayoría de estos aparatos están constituidos por elementos como: metales, vidrios y plásticos que por el tipo de inspección en primera instancia no parecerían constituirse en un problema salvo por el espacio que ocuparían en los vertederos al ser eliminados.

Según Castellanos (2005), los desechos electrónicos generalmente están constituidos por: polímeros en un 30\% (plásticos), óxidos refractarios en un 30\% (cerámicos) y por metales en un $40 \%$.

A su vez a los metales presentes en la chatarra electrónica se los puede dividir en dos grupos:

1. Metales Básicos

a. Cobre del $20 \%$ al $50 \%$

b. Hierro del $8 \%$ al $20 \%$ 

c. Níquel del $2 \%$ al $5 \%$
d. Estaño del $4 \%$ al 5\%
e. Plomo aproximadamente $2 \%$
f. Aluminio del $2 \%$ al $5 \%$
g. Zinc del $1 \%$ al $3 \%$

2. Metales preciosos
a. Oro de $170 \mathrm{~g}$ a $850 \mathrm{~g}$ aproximadamente el $0.1 \%$
b. Plata de $198 \mathrm{~g}$ a $1698 \mathrm{~g}$ aproximadamente el $0.2 \%$
c. Paladio de $3 \mathrm{~g}$ a $17 \mathrm{~g}$ aproximadamente el $0.005 \%$.

Un análisis más profundo, revela por ejemplo:

1. Que entre los metales, no solamente se encuentran los ya mencionados, sino también el bismuto y los denominados metales pesados como el arsénico, el cadmio, el cromo, el mercurio, el plomo y el selenio.

2. Diversos tipos de plásticos con o sin retardante de llama.

3. La presencia de vidrio en aparatos visualizadores como las modernas pantallas de cristal líquido o los tradicionales tubos de rayos catódicos.

4. La presencia de dispositivos como acumuladores, pilas y baterías, capacitores, resistores, relés, sensores, conductores, circuitos impresos, medios de almacenamiento de datos, elementos de generación de luz, sonido y calor, etc.

\section{COMPONENTES PELIGROSOS EN LOS DISPOSITIVOS ELÉCTRICOS Y ELECTRÓNICOS.}

Entre los componentes de los elementos eléctricos y electrónicos, se encuentran sustancias y materiales tóxicos, como los metales pesados, los Bifenilos Policlorados, los Éteres Bifenílicos Polibromados y materiales que al incinerarse en condiciones inadecuadas son precursores de la formación de otras sustancias tóxicas como las dioxinas y los furanos, todas estas ambientalmente problemáticas.

Por esta razón, la eliminación de los RAEE, se viene constituyendo en un grave problema ya que por ejemplo: "cada monitor de computadora o pantalla de televisor contiene entre 2 y 8 libras de plomo" 
(Martínez, 2008). "Ese plomo lo absorbemos cuando esa pantalla va a un tiradero en el campo, en el suelo, a lo largo de los años se desintegra y, cuando llueve, pasa a los mantos freáticos. Eventualmente, a largo plazo, tomaremos agua de ahí, entonces nos llegará el plomo al organismo” (Daniel, 2008).

De la misma manera al acumularse al aire libre, los demás componentes peligrosos, contaminarán el suelo, el agua y el aire seguramente provocando en poco tiempo el aparecimiento de problemas graves de salud.

A continuación y con la ayuda de las Fichas Internacionales de Seguridad Química, se hace una caracterización de los componentes peligrosos, con el propósito de aportar con argumentos, del porqué los RAEE se están constituyendo en un gran problema para la humanidad.

\section{Plomo}

Se presenta como plomo u óxido de plomo, en soldaduras, en placas de baterías, en los tubos de rayos catódicos de los computadores y televisores. Se calcula que un televisor contiene cerca de $2 \mathrm{~kg}$.de plomo y un computador personal cerca de $0,4 \mathrm{~kg}$.

Se puede absorber por inhalación del aerosol y por ingestión. La evaporación a $20^{\circ} \mathrm{C}$ (temperatura ambiente) es despreciable; sin embargo, se puede alcanzar rápidamente una concentración nociva de partículas en el aire. La exposición de corta duración puede causar efectos en el tracto gastrointestinal, sangre, sistema nervioso central y riñón, dando lugar a cólicos, shock, anemia, daño renal y encefalopatías. La exposición puede producir la muerte. Los efectos pueden aparecer de forma no inmediata. Se recomienda vigilancia médica. La exposición prolongada o repetida puede afectar al tracto gastrointestinal, sistema nervioso, sangre, riñón y sistema inmunológico, dando lugar a cólicos graves, parálisis muscular, anemia, cambios en la personalidad, retardo en el desarrollo mental, nefropatías irreversibles. Puede causar retardo en el desarrollo en los recién nacidos. Posibilidad de efectos acumulativos.

Esta sustancia puede ser peligrosa para el ambiente; debería prestarse atención especial al aire y al agua. En la cadena alimenticia referida a los seres humanos tiene lugar bioacumulación, concretamente en vegetales y organismos acuáticos, especialmente en los peces.

\section{Mercurio}


Se estima que más del 90\% del mercurio de los RAEE proceden de las pilas y sensores de posición, aunque también se lo encuentra aunque en pequeñas cantidades en los relés y tubos fluorescentes.

Se lo puede absorber por inhalación, a través de la piel y también como vapor. Por evaporación de esta sustancia a $20^{\circ} \mathrm{C}$ se puede alcanzar muy rápidamente una concentración nociva en el aire. Por exposición de corta duración, la inhalación del vapor puede originar neumonitis. El mercurio puede causar efectos en el riñón y en el sistema nervioso central. Los efectos pueden aparecer de forma no inmediata. Se recomienda vigilancia médica. Por exposición prolongada o repetida, la sustancia puede afectar al sistema nervioso central y al riñón, dando lugar a inestabilidad emocional y psíquica, temblores, alteraciones cognitivas y del habla. Peligro de efectos acumulativos. La experimentación animal muestra que esta sustancia posiblemente cause efectos tóxicos en la reproducción humana.

En cuanto al medio ambiente, esta sustancia es muy tóxica para los organismos acuáticos. En la cadena alimenticia referida a los seres humanos tiene lugar bioacumulación, concretamente en los peces.

\section{$\underline{\text { Cadmio }}$}

Se estima que más del 90\% del cadmio de los RAEE procede de las pilas recargables, también se lo encuentra en determinados componentes de los circuitos impresos y es utilizado como estabilizador en el PVC.

$\mathrm{Al}$ cadmio se lo puede absorber por inhalación del aerosol y por ingestión. La evaporación a $20^{\circ} \mathrm{C}$ es despreciable; sin embargo, se puede alcanzar rápidamente una concentración nociva de partículas en el aire. Por efecto de una exposición de corta duración la sustancia irrita los ojos y el tracto respiratorio. La inhalación del humo puede originar edema pulmonar y fiebre de los humos metálicos. Los efectos pueden aparecer de forma no inmediata. Se recomienda vigilancia médica. Los pulmones pueden resultar afectados por la exposición prolongada o repetida a las partículas de polvo. La sustancia puede afectar al riñón, dando lugar a proteinuria y disfunción del riñón. Esta sustancia es probablemente carcinógena para los seres humanos.

\section{$\underline{\text { Bario }}$}


Se lo utiliza generalmente en los paneles frontales de los tubos de rayos catódicos con el propósito de proteger de la radiación a los usuarios.

El Bario se puede absorber por ingestión. La sustancia irrita los ojos, la piel y el tracto respiratorio. "Estudios han demostrado, que ciertas exposiciones al bario han causado aumento y endurecimiento del cerebro, flaqueza muscular, daños al corazón y al hígado” (BAN, 2002).

\section{$\underline{\text { Cromo }}$}

El cromo metálico de la forma cromo 0, se encuentra presente en los elementos ferrosos o acerados pues es un componente de este. El cromo VI conocido como hexavalente se usa en el cromado en las tinturas y pigmentos.

El cromo se puede absorber por inhalación del aerosol y por ingestión. La evaporación a $20^{\circ} \mathrm{C}$ es despreciable; sin embargo, se puede alcanzar rápidamente una concentración nociva de partículas en el aire cuando se dispersa. El contacto prolongado o repetido puede producir sensibilización de la piel.

"La Organización Mundial de la Salud (OMS) ha determinado que el cromo hexavalente es carcinógeno en seres humanos; en el mismo sentido el Department of Health and Human Services (DHHS) de los Estados Unidos ha determinado que ciertos compuestos de cromo hexavalente producen cáncer en seres humanos y, la Environmental Protection Agency de Estados Unidos ha establecido que el cromo hexavalente en el aire es carcinogénico en seres humanos" (Roman).

\section{$\underline{\text { Arsénico }}$}

Se lo encuentra en los tubos de rayos catódicos antiguos. El Arsénico se puede absorber por inhalación del aerosol, a través de la piel y por ingestión. La evaporación a $20^{\circ} \mathrm{C}$ es despreciable; sin embargo, se puede alcanzar rápidamente una concentración nociva de partículas en el aire. Debido a la exposición de corta duración la sustancia irrita los ojos, la piel y el tracto respiratorio. La sustancia puede causar efectos en el sistema circulatorio, sistema nervioso, riñón y tracto gastrointestinal, dando lugar a convulsiones, alteraciones renales, graves hemorragias, pérdida de fluidos y electrolitos, shock y muerte. La exposición puede producir la muerte. Los efectos pueden aparecer de forma no inmediata. Se recomienda vigilancia médica. El contacto prolongado o repetido con la piel puede producir dermatitis. El contacto prolongado o repetido puede producir sensibilización de la piel. La sustancia puede afectar 
a las membranas mucosas, piel, riñón e hígado, dando lugar a neuropatías, desórdenes en la pigmentación, perforación del tabique nasal y alteraciones tisulares. La sustancia es carcinógena para los seres humanos.

El Arsénico es tóxico para los organismos acuáticos. Se aconseja firmemente impedir que el producto químico se incorpore al ambiente.

\section{$\underline{\text { Selenio }}$}

Generalmente está presente en los tableros de circuitos como rectificador de suministro de energía.

El Selenio se puede absorber por inhalación, a través de la piel y por ingestión. La evaporación a $20^{\circ} \mathrm{C}$ es despreciable; sin embargo, se puede alcanzar rápidamente una concentración nociva de partículas en el aire por dispersión. Por efecto de una exposición de corta duración la sustancia irrita los ojos y el tracto respiratorio. La inhalación del polvo puede originar edema pulmonar. La inhalación del humo puede originar síntomas de asfixia, escalofríos, fiebre y bronquitis. Los efectos pueden aparecer de forma no inmediata. El contacto prolongado o repetido con la piel puede producir dermatitis. La sustancia puede afectar al tracto respiratorio, al tracto gastrointestinal y a la piel, dando lugar a náuseas, vómitos, tos, coloración amarilla de la piel, pérdida de uñas, aliento aliáceo y alteraciones dentales.

\section{$\underline{\text { Los Bifenilos Policlorados (PCB) }}$}

Poseen magnificas propiedades dieléctricas, y de longevidad, no son inflamables y son resistentes a la degradación térmica y química. Los (PCB) tienen 12 congéneres a los que la Organización Mundial de la Salud ha asignado factores de equivalencia de toxicidad por su comportamiento parecido en este aspecto al de la dioxina.

Antes de ser prohibidos eran utilizados en la fabricación de: transformadores eléctricos, condensadores eléctricos, reactancias de lámparas, interruptores eléctricos, relés y otros accesorios, cables eléctricos, motores eléctricos y electroimanes, además era utilizado como plastificante en cloruro de polivinilo, neopreno y otras resinas artificiales.

\section{$\underline{\text { Los Terfenilos Policlorados (PCT) }}$}


Poseen propiedades físicas y químicas muy parecidas a las de los PCB por lo que se les utilizaron en las mismas aplicaciones, son prácticamente insolubles en agua y muy resistentes a la degradación, además de ser menos volátiles que los PCB. Las cantidades que se utilizaron en aplicaciones eléctricas fueron muy pequeñas.

\section{$\underline{\text { Los Bifenilos Policromados (PBB) }}$}

Son sustancias sólidas o cerosas a temperatura ambiente. Son prácticamente insolubles en agua y sumamente resistentes a la degradación.

Los PBB se utilizaban fundamentalmente como retardadores de llama. Se añadían al plástico de acrilonitrilo butadieno estireno, a las pinturas, lacas y a la espuma de poliuretano.

Los RAEE que contengan o estén contaminados con PCB, PCT o PBB están constituidos por elementos como: condensadores, disyuntores, cables eléctricos motores, electroimanes, interruptores, transformadores, reguladores de voltaje, disolventes, selladores, pinturas, fluidos dieléctricos y plásticos.

\section{$\underline{\text { Retardantes de llama }}$}

Son químicos que se agregan a los componentes plásticos (carcasas) en el caso de los equipos electrónicos, con el propósito de evitar que el fuego se esparza con facilidad. Los más utilizados son los siguientes:

1. Éteres de Polibromodifenilos (PBDEs)

Son químicos ambientalmente persistentes, algunos altamente bioacumulativos y con capacidad de interferir en el desarrollo normal del cerebro de los animales. Existe la sospecha de que varios de estos PBDSs son disruptores endócrinos y que presentan facilidades para interferir a las hormonas relacionadas con el crecimiento y el desarrollo sexual. Existen estudios en los cuales se ha comprobado que afectan al sistema inmunológico.

2. Tetrabromobisfenol - A (TBBPA) 
Se ha comprobado que pueden interferir con las hormonas tiroideas, que tienen efectos sobre el crecimiento y el desarrollo, se cree además que presentan efectos potenciales sobre otros sistemas hormonales, sobre el sistema inmunológico, el hígado y los riñones.

3. Fosfato de Trifenilo (TPP)

Es sumamente tóxico para la vida acuática, es un fuerte inhibidor de un sistema de enzimas vitales en la sangre humana. Puede causar dermatitis por contacto en algunas personas y es un posible disruptor endócrino.

\section{IMPACTO AMBIENTAL}

Como ya se mencionó anteriormente este tipo de desecho es el de mayor crecimiento en los últimos años, y crece de manera paralela al aumento en el consumo o utilización de nuevos y modernos aparatos eléctricos y electrónicos.

Por poner tan solo un ejemplo de este crecimiento vertiginoso en la tabla 1, se presenta las ventas anuales en seis países representativos de América Latina y el Caribe de PC's que en pocos años se convertirán en basura.

Tabla 1. Ventas anuales de PCs en los países más representativos de Latinoamérica y el Caribe entre 1983 y el 2008

\begin{tabular}{|l|r|r|r|r|r|r|r|r|r|r|}
\hline País & $1993-1999$ & 2000 & 2001 & 2002 & 2003 & 2004 & 2005 & 2006 & 2007 & 2008 \\
\hline Argentina & 3247 & 880 & 660 & 110 & 420 & 675 & 1200 & 1226.7 & 1324.5 & 1404.4 \\
\hline Brasil & 14930.3 & 2989.6 & 3364.9 & 3310.4 & 3537.9 & 4280.2 & 5384.4 & 6031.5 & 6633.1 & 7197.8 \\
\hline Chile & 1883 & 388.1 & 389.7 & 399.9 & 460.9 & 615.9 & 745.4 & 816.4 & 873.7 & 917.2 \\
\hline Colombia & 1904.6 & 294 & 313.3 & 379 & 446.2 & 526.5 & 662.3 & 760.2 & 852.6 & 937.1 \\
\hline México & 9128.4 & 1822 & 2020.5 & 1921.5 & 1889.4 & 2383.5 & 2659.7 & 3114.4 & 3355.4 & 3698.6 \\
\hline Venezuela & 1349.8 & 285.4 & 294.7 & 231.9 & 174.9 & 276.2 & 383.2 & 452.1 & 519 & 572.4 \\
\hline Total & 32443.1 & 6659.1 & 7043.1 & 6352.7 & 6929.3 & 8757.3 & 11035 & 12401.3 & 13558.3 & 14727.5 \\
\hline En miles de unidades & & & & & & & & \\
\hline
\end{tabular}

De acuerdo a la descripción que se hizo de los principales componentes de estos aparatos y equipos, su eliminación al dejar de ser utilizados provocan los siguientes efectos:

1. Por su peso y volumen ocupa gran cantidad de espacio al ser enviados como basura convencional a los llamados vertederos. Al no gestionar técnicamente su eliminación total, el gran peligro 
constituye la contaminación del suelo, del aire y del agua con sustancias que tóxicas para la salud humana que inclusive pueden resultar de la interacción con el medio ambiente que también resultará contaminado acarreando consecuencias sumamente negativas.

2. La presencia de muchas personas en los vertederos, gestionando de manera antitécnica este tipo de residuos, con el propósito de obtener de ellos plástico, metales, vidrio y otros materiales con el grave riesgo de resultar afectados por las sustancias toxicas propias de estos aparatos o de las que se producen como resultado de la interacción con el medio ambiente.

3. La imposibilidad de un reciclado fácil, rentable, seguro para los seres humanos y de baja contaminación para el medio ambiente, provoca grandes consumos de energía y recursos naturales. "Y la situación empeora si se considera el uso de los recursos en el sector electrónico. Fabricar un PC con una pantalla plana de 17 pulgadas, demanda $240 \mathrm{~kg}$ de combustible fósil, $22 \mathrm{~kg}$ de productos químicos 1500 litros de agua. Otro ejemplo: una planta de chips consume 7 millones de litros de agua cada día" (Duery, 2007).

Como una consecuencia en el crecimiento de la tasa de renovación tecnológica y la gran acumulación de equipos que han cumplido su ciclo de vida, los aparatos electrónicos y sus residuos crecen más rápido que la implementación de nuevos equipos y mucho más que la conciencia ambiental junto con su normativa y los programas eficaces para su manejo que se fomenten.

En la figura 1 se muestra este comportamiento con lo que se llama la "Ley de la basura electrónica" (Prince, 2006). 


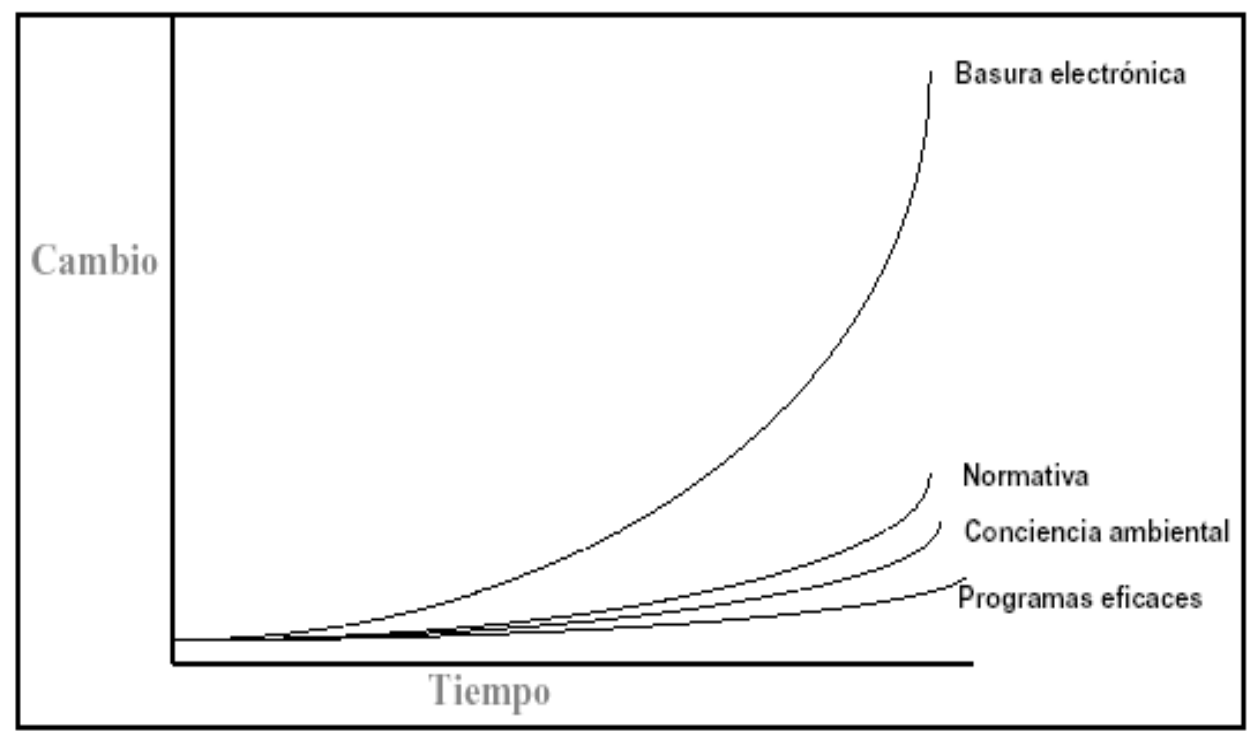

Figura 1. Ley de la Basura Electrónica

\section{GESTION DE LOS RAEE EN LOS PAISES EN DESARROLLO.}

Según la Organización Ambientalista Basel Action Network (BAN), con sede en la ciudad estadounidense de Seattle "Unos 500 contenedores cargados con un volumen equivalente a 400000 monitores de computadora o 175000 aparatos de televisión ingresan a Lagos capital comercial de Nigeria al mes" (Olucoya, 2008). A Lagos ingresan con gran facilidad artículos importados de segunda mano por su bajo costo que en su mayoría son descartados poco tiempo después, creándose un gran problema ambiental y de salud para los habitantes que se encuentran cerca de los vertederos que se forman, debido que la gran mayoría de estos desechos son quemados al aire libre, emitiendo partículas tóxicas.

"En un informe realizado en febrero del 2002 por los grupos BAN (Basel Action Network) y SVTC (Silicon Valley Toxics Coalition), apoyados por Greenpeace China, Toxics Link India y SCOPE de Pakistán, existen denuncias de que cerca del 80\% de los residuos de aparatos electrónicos generados en los Estados Unidos serán exportados para China, Pakistán e India para ser reciclados, donde las tareas se realizaban en malas condiciones ambientales y sin ninguna precaución a la salud de los trabajadores" (Casia).

"Según un artículo de la revista Time, la gran mayoría de la basura de equipos electrónicos termina en países como China, India y Nigeria donde se encargan de su despacho” (Martínez, 2008). 
En el Ecuador, no existe una política para gestionar este tipo de residuos ni de parte del Ministerio del Ambiente, ni de la Dirección de Medio Ambiente del Distrito Metropolitano de Quito.

Se pudo conocer de manera preliminar que existen dos gestores en Quito: La Fundación Hermano Miguel y Servercompu, que desarman y extraen los componentes que pueden ser utilizados localmente, para según dijeron el resto exportar a Europa en donde existe la tecnología apropiada para seguir reciclándolos o eliminarlos por completo. Pero pocos conocen de su existencia y generalmente lo que se ve es que los habitantes abandonan en la vía pública los aparatos cuando ya no le son útiles los mismos que terminan en los botaderos de basura o en las casas de gente muy humilde que cree que podrá hacerlos funcionar.

Por lo tanto es en los países en desarrollo como el nuestro que debe existir una gran preocupación por conocer las alternativas de gestión de este tipo de desechos de manera técnica a fin de no comprometer la calidad del ambiente y la salud de los seres humanos.

\section{GESTIÓN DE LOS RAEE, BUSCANDO EL RECICLAJE}

Con el propósito de gestionar los desechos electrónicos, la UNU, el Programa de Medio Ambiente de la ONU, la Agencia de Protección Ambiental de Estados Unidos, algunas Universidades del mundo y empresas como Dell, Microsoft, HP y Philips, crearon la iniciativa "Solucionar el problema de la ebasura". "Este proyecto busca, entre otras cosas, homogenizar los procesos de reciclado en el mundo con el fin de que los componentes valiosos sean recuperados" (Aguayo, 2007).

Una de las principales compañías dedicadas a esta actividad es Hong Kong Recycling Company, empresa que puede llevar hasta 70000 libras en solo furgón, puede pagar de 20 a 25 centavos por libra.

Los teléfonos, computadoras, televisores y demás aparatos eléctricos y electrónicos, generalmente pasan por un proceso de desarmado manual, seleccionando los componentes que aún pueden ser utilizados en la fabricación de nuevos productos, de la industria electrónica principalmente.

"Según Carlos Arizaga gerente de Seguridad y Medio Ambiente de TCG en México y Brasil: Al residuo se le da un valor en la cadena productiva por lo que automáticamente deja de ser residuo, ya que se le da otro uso y no va a los rellenos sanitarios. Desde el punto de vista ambiental, al entregar los componentes a otros procesos, colaboramos a que la naturaleza no sea explotada" (Daniel, 2008). 
Actualmente existen empresas en Bélgica, Japón, China, Singapur y Estados Unidos que reciben los remanentes de aparatos electrónicos procedentes de cualquier parte del mundo para reciclar y elaborar otros productos.

Como ejemplos en este tipo de actividad vale la pena mencionar los siguientes:

1. Aproximadamente hace 19 años el consorcio transnacional Hewlett-Packard, comenzó el reciclado de componentes electrónicos a través de la corporación transnacional Micro Metallics que inicialmente procesó aproximadamente 18000 toneladas anuales.

2. En el año 2002, Micro Metallics, abrió una novedosa estación de reciclaje en Roseville, EE.UU. Ellos transportan productos entre otros procedentes de: Hewlett-Packard, Compaq, Xerox, Digital Equipment Corp y Sol Microsystems. Movilizan diariamente veinte semirremolques desde todos los estados los componentes reciclables.

Las computadoras que resultan de actualizaciones de oficinas y que llegan "intactas", se limpian se prueban y se certifican para la reventa en el mercado de uso, las restantes se desmantelan para su reciclado.

Los plásticos de las computadoras inutilizables, se clasifican de acuerdo a sus características físicas y químicas y se envían a los fabricantes, quienes los funden para elaborar sus nuevos productos.

"El reciclaje de la chatarra electrónica ha desarrollado algunas técnicas muy refinadas que han sobrepasado el de la industria de reciclaje de los vehículos” (Castellanos, 2005).

\section{RESULTADOS Y DISCUSIÓN}

La industria de aparatos y equipos eléctricos y electrónicos, es una de las de mayor crecimiento en la actualidad, producida por la demanda cada vez más grande en todos los lugares del planeta por las facilidades que prestan en el desenvolvimiento y desarrollo de todas las actividades humanas. 
A la par, al ser declarados como elementos inservibles, declaración que cada vez se la hace en períodos de tiempo más cortos, pues esa es la característica de duración que está prevaleciendo en este tipo de aparatos, crece el problema serio de cómo deshacerse de esta de basura, desecho o residuo.

Existen ya consideraciones ambientales por el impacto negativo que están causando y que se acentuaría si no se implementan de manera técnica procesos para fabricarlos, para identificarlos, ubicarlos, recolectarlos, transportarlos, desarmarlos, reutilizar partes con las cuales se pueden elaborar nuevos productos, reciclar componentes, y por último eliminarlos sin contaminar el medio ambiente ni afectar a la salud humana.

Como es lógico para disminuir los efectos del problema, lo ideal sería consumir el mínimo de elementos que resulten tóxicos en cualquier etapa de la existencia del aparato y al mismo tiempo buscar reemplazar estos por elementos más amigables para el ambiente y el ser humano.

De todas maneras el problema está presente y afectando ya sea de manera directa o indirecta tanto a países industrializados como en vías de desarrollo, por lo que las soluciones tienen que ser integrales, normalizadas y acogidas por la humanidad entera antes de que se convierta en algo irreversible o demasiado costoso su solución o remediación.

Se está avanzando en el reciclaje como parte de la solución, pero actualmente el $90 \%$ de los equipos acaban en los vertederos después de haber sido abandonados en la calle o lanzados sin ningún criterio en contenedores que los transportarán.

Vale la pena recalcar que de este tipo de basura, cerca del 50\% es metal que puede ser extraído y reprocesado, el resto es plástico y vidrio que puede seguir el mismo camino y que por lo tanto puede adquirir valor económico, beneficiando a quienes de manera técnica la gestionen.

Como una vía para frenar el crecimiento de la basura electrónica se ha presentado la iniciativa "Solucionar el problema de la e-basura" (STEP, por sus siglas en inglés), por el Programa del Medio Ambiente de Naciones Unidas (PNUD), universidades de los cinco continentes y empresas como Dell, Microsoft, Hewlett Packard y Philips, entre muchas otras con el propósito de estandarizar el reciclado en el mundo, extender la vida útil de los aparatos y homogenizar las legislaciones. 


\section{CONCLUSIÓN}

Por lo que es imperioso que en el país se comience a crear conciencia de la generación de este tipo de basura, para que desde las instancias de poder correspondientes se implementen medidas probadas, que tiendan a disminuir tanto la generación como la gestión antitécnica y desorganizada que trae consigo la contaminación del medio ambiente perjudicando como siempre al ser humano.

\section{BIBLIOGRAFÍA}

Aguayo Olivia. (2007). Lucha la ONU contra e-basura. Reforma. México D.F., México.

Angela Cassia Rodrigues. El Crecimiento de los Residuos de Aparatos Eléctricos y Electrónicos fuera de uso: El Impacto Ambiental que presentan. http://www.bvsde.paho.org/bvsaidis/mexico2005/cassia.pdf

Castellanos Néstor Alonso. (2005). La chatarra electrónica, la contaminación ambiental y su efecto económico. XVI Forum de Ciencia y Tecnología. La Habana Cuba.

Daniel Santiago. (2008) El camino que sigue tu basura electrónica. El Norte. México D.F., México.

Duery A. Lilian. (2007). La basura electrónica crece el triple que la domiciliaria. El Mercurio de Chile. Marzo12.

Martinez Rivera Carlos. (2008). A China la basura electrónica. El Nuevo Día de Puerto Rico. Julio 20.

Prince Alejandro. (2006). Presentación en el primer Taller de Reciclaje SUR-IDRC, Brasilia.

Olukoya Sam. (2008). Ambiente- Nigeria: Bajo la Basura Electrónica. Noticias en Español. Enero28.

Román Moguer Guillermo.(2007). Diagnóstico sobre la generación de basura electrónica en México. 